\title{
Comparison of Mature, Immature, and Neurotic Defense Mechanisms in Veterans and Normal People
}

\section{ART ICLE INF O}

\section{Article Type}

Descriptive Study

\section{Authors}

Kazemian R. ${ }^{1} M A$,

Abolmaali Alhosseini Kh.* PhD

\section{A B S T R A C T}

Aims The stresses and fears experienced by veterans affect their use of defense mechanisms. The aim of this study was to compare the mature, immature, and neurotic defense mechanisms in veterans and normal people.

Materials \& Methods In the present causal-comparative retrospective study, the statistical population included veterans supported by the Martyr Foundation and Issargaran affairs in Tehran 1395, and normal people over the age of 25 living in Tehran. In this study, 40 veterans were selected from Sarallah Rehabilitation Hospital, Sadr Psychiatric Hospital, and Niyayesh Psychiatric Hospital and 40 normal people were selected from the husbands of female students of the Center for Behavioral Cognition by convenience sampling method. The Defense Style Questionnaire was used as a research tool and the data were analyzed by SPSS 22 software through multivariate analysis of covariance and Bonferonni post hoc test.

Findings By controlling the effect of age, there was a significant difference in the use of various defense mechanisms between veterans and normal people $(\mathrm{p}<0.01)$. The difference between the mean scores of using the mature and immature defense mechanisms was significant in the two groups $(\mathrm{p}<0.01)$. Veterans used more immature defense mechanisms than normal people and used less mature defense mechanisms. There was no significant difference between the mean scores of neurotic defense mechanisms in the two groups ( $p>0.05)$.

Conclusion Veterans use more mature and immature defense mechanisms than normal people, but they do not differ in the use of neurotic defense mechanisms.

Keywords Defense Mechanisms; Mature Defense Mechanisms; Immature Defense Mechanisms;
Neurotic Defense Mechanisms; Veterans

\section{I T A T I O N L I N K S}

[1] Effect of music-therapy on depression ... [2] A survey on various dimensions ... [3] PTSD symptoms and suicide risk ... [4] Prevalence of mental disorders in ... [5] Mental health status of amputee veterans ... [6] Comparison of stress and social support between veterans ... [7] Study of health related quality of life in posttraumatic ... [8] Fatigue and sleepiness determine respiratory quality ... [9] Quality of life in chemical war victims with sever pulmonary ... [10] Type of injury and quality of life among ... [11] Physical and social factors determining ... [12] Consideration of chronic pain and current prosthetic ... [13] Genitourinary disease in war related ... [14] Epidemiology of mental health status ... [15] The survey of quality of life in spinal cord injured people ... [16] Link between early maladaptive schemas ... [17] Linking students' emotions and academic ... [18] Defense mechanisms, negative emotions ... [19] Defenses: How the mind ... [20] Adaptive midlife defense mechanisms and ... [21] Theory and practice of counseling and ... [22] Defense mechanisms in psychology today ... [23] The comparison of defense mechanisms of the ... [24] Psychology: The science of ... [25] Adaptive mental mechanisms, their role in ... [26] Psychology ... [27] Comparison of the type of coping mechanisms ... [28] The defense style ... [29] Defense mechanisms in soldiers during the ... [30] The role of defense mechanisms in the modulation of anger ... [31] Defense mechanisms in patients with major ... [32] The role of social support and coping strategies on mental ... [33] Cognitive-behavioral coping strategies associated ... [34] The level and type of irrational beliefs ... [35] Theoretical and practical foundations of research ... [36] Validity and reliability of the defensive styles questionnaire in Iranian ... [37] Epidemiology of substance abuse in war survivors ... [38] An examination of alcohol risk profiles and ... [39] A study of the relationship between mental ... [40] Comparison of defense styles \& defense mechanisms of patients with obssesive-compulsive disorder ... [41] Longitudinal assessment of mental health ... [42] What is the relationship between alexithymia ... [43] Predicting defense mechanisms based on big five personality traits among university ...

\section{Article History}

Received: October 20, 2017

Accepted: April 28, 2018

ePublished: October 11, 2018 
كاهش سطح فعاليت فيزيكى، بيمارىهاى عضلانى- اسكلتى و

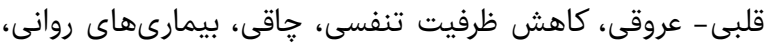

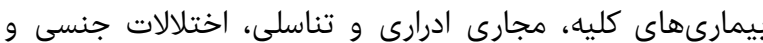

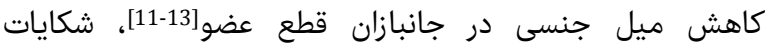

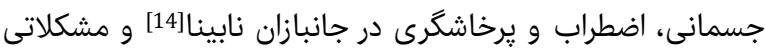

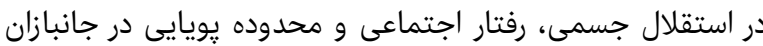
داراى ضايعه نخاعى[15] مورد بررسى قرار گرفتئه استئ.

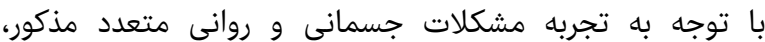

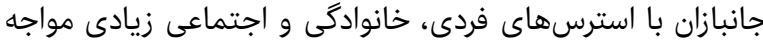

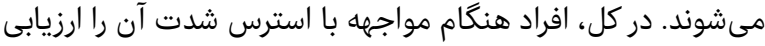

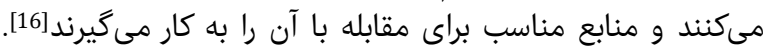

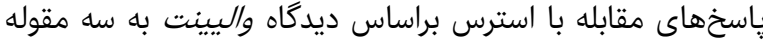

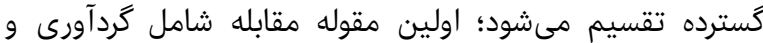

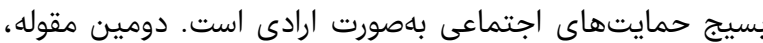

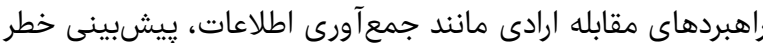

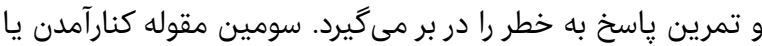

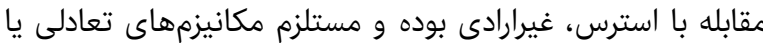

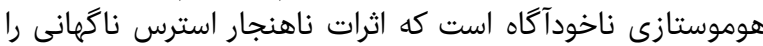

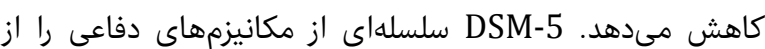
رشدنايافته به رشديافته ارايه مىدهد مهد [17].

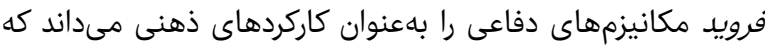
از افراد در برابر اضطراب زياد ناشى از رويدادهاى استرسزاي داى بيرونى إنى

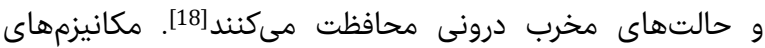

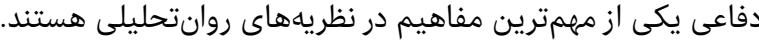

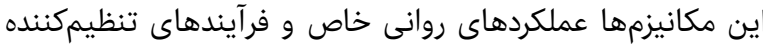

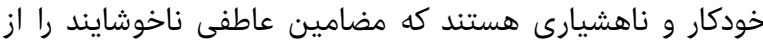

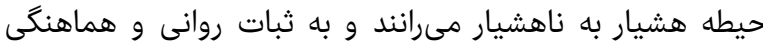

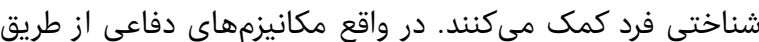

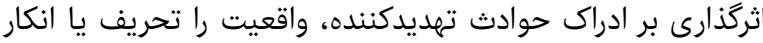

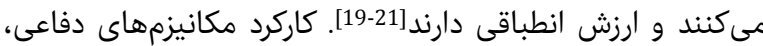

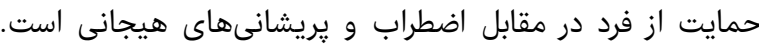

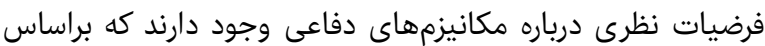

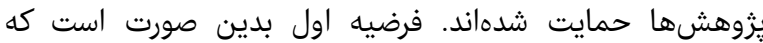

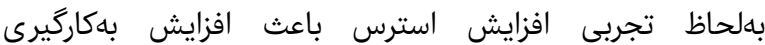

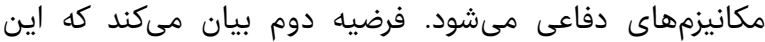

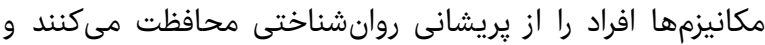

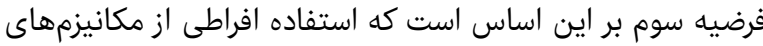

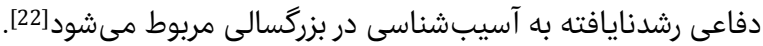

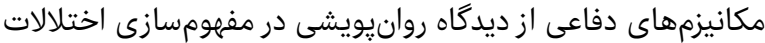

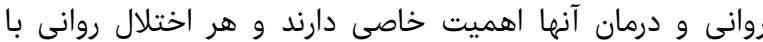

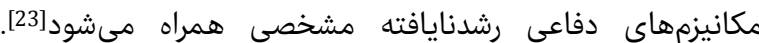

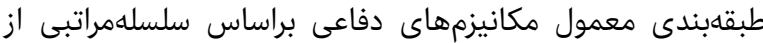

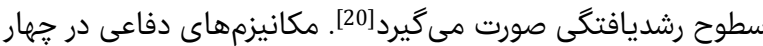

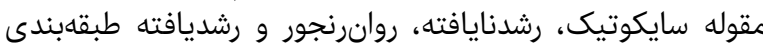

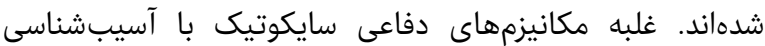

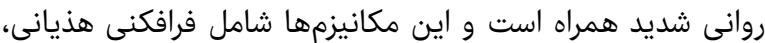

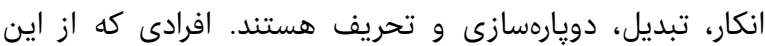

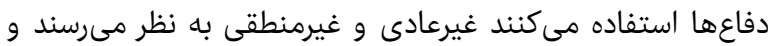

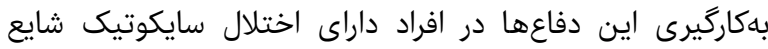

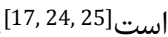

مكانيزمهاى دفاعى رشديافته به منزله شيوههاى مواجهه انطباقى،

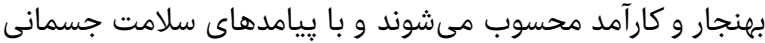

دوره •ا، شماره F، ياييز Vqسו
مقايسه مكانيزمهاى دفاعى رشديانيافته، رشدانايافته و روانرنجورانه در افراد عادى و جانباز داعى رسائه

MA وضا كاظميان

كروه روانشناسى، دانشكده روانشناسى، واحد رودهن، دانشي، دانشكاه آزاد اسلامى،

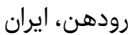

PhD * خديجه ابوالمعالى الحسينى

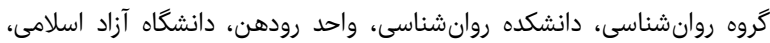

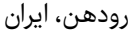

حكيده

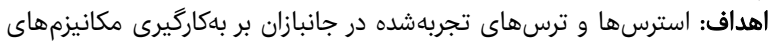

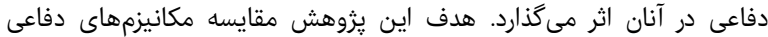

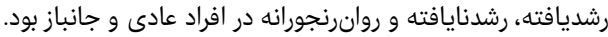

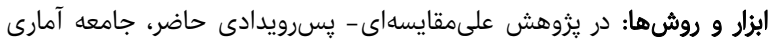

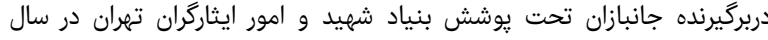

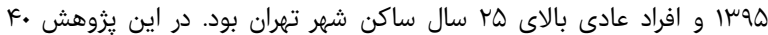

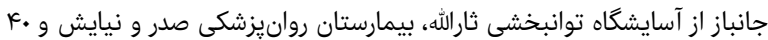

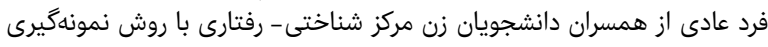

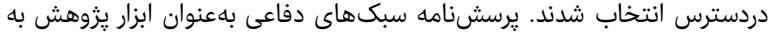

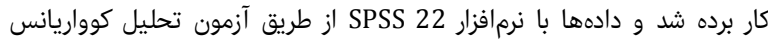

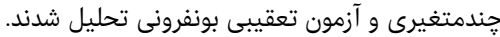

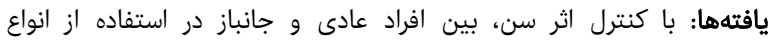

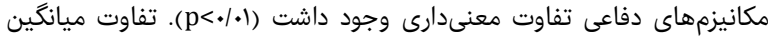

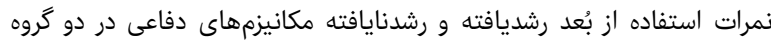

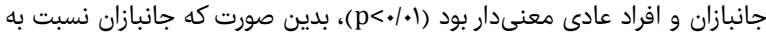

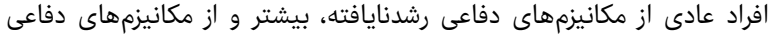

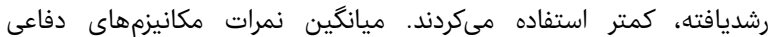

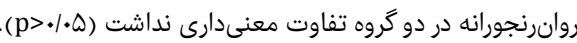

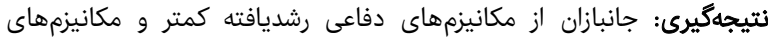

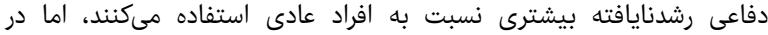

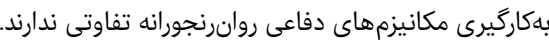

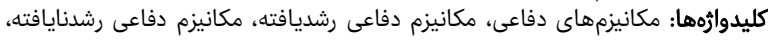
مكانيزم دفاعى روانرنجورانه، جانباز

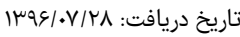

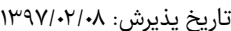

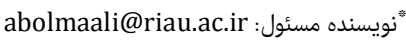

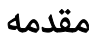

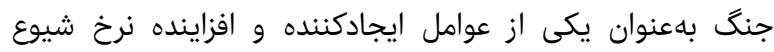

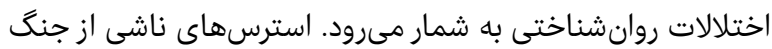

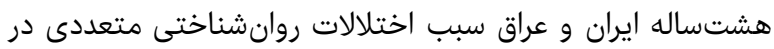

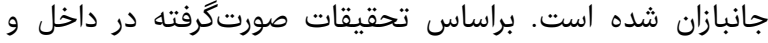

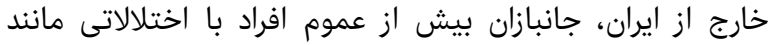

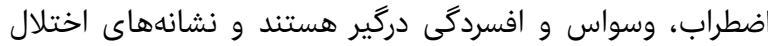

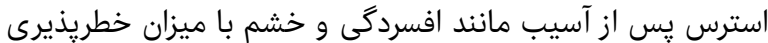

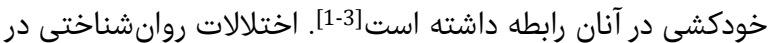

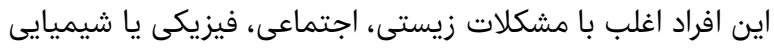

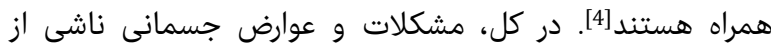

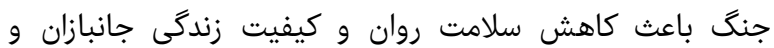

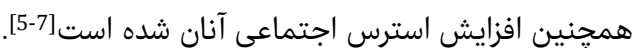

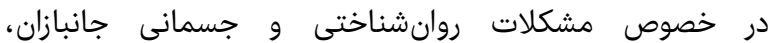

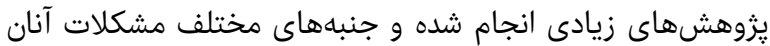

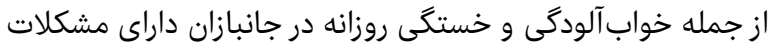

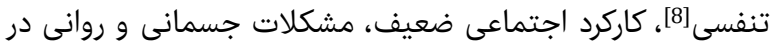

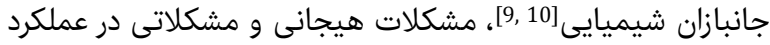

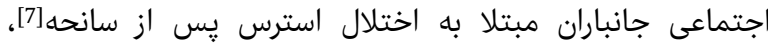




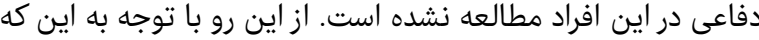

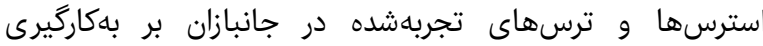

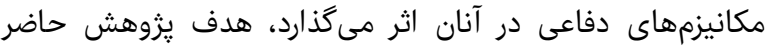

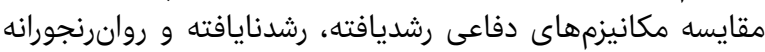

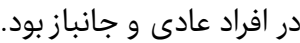

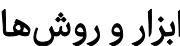

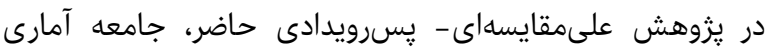

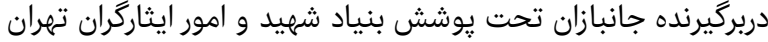

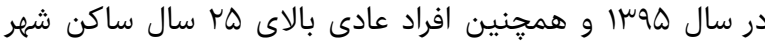

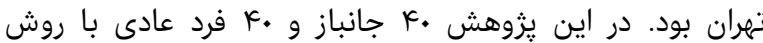

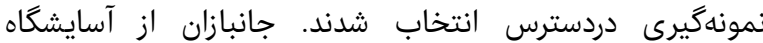

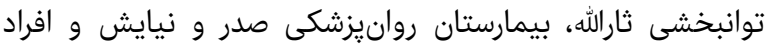

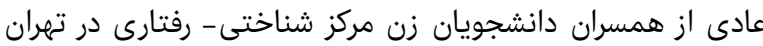

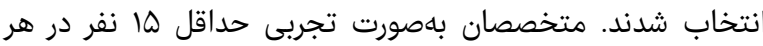

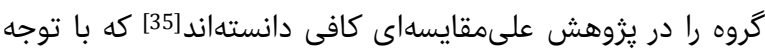

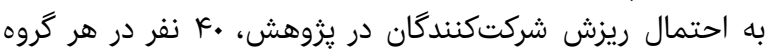

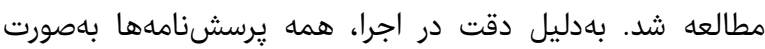

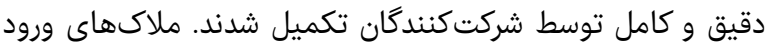

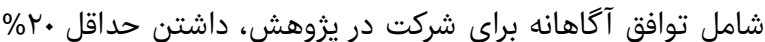

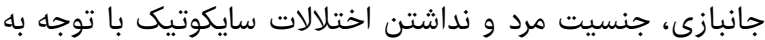

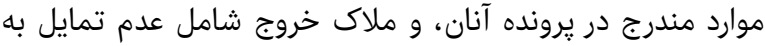

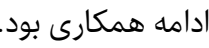

يرسشنامه سبكهاى دفاعى (DSQ): اين يرسشنام:امه در سال

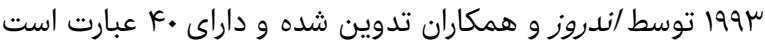

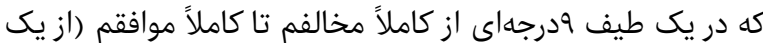

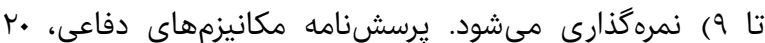

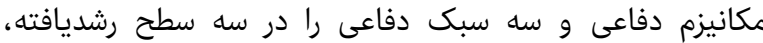

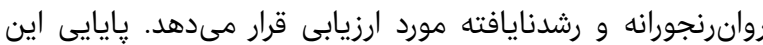

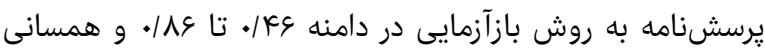

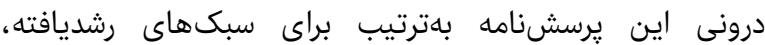

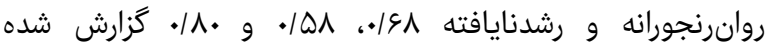

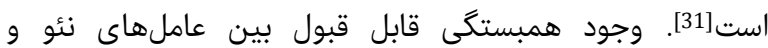

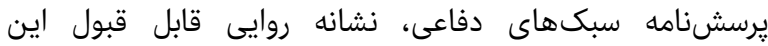

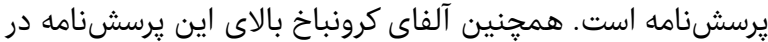

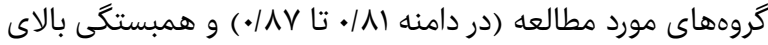

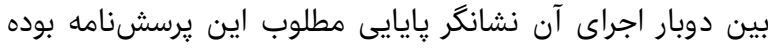

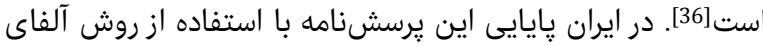

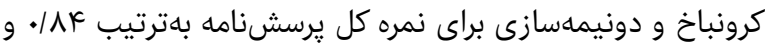

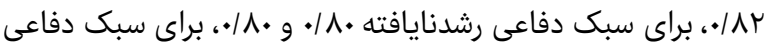

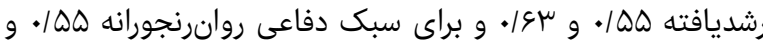

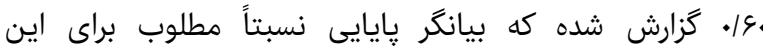

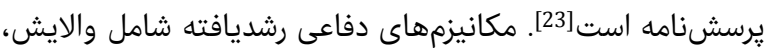

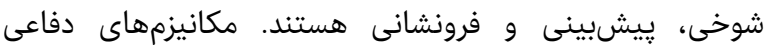

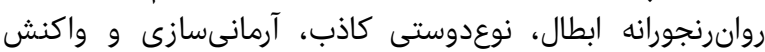

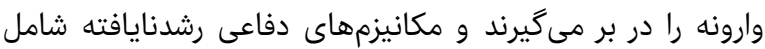

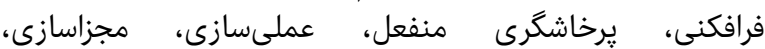

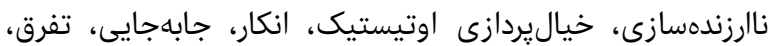

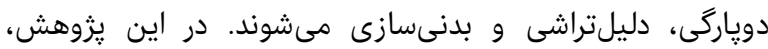

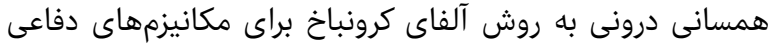

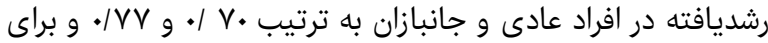

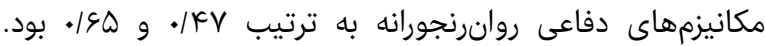

و روانى در ارتباط هستند. افرادى كه از مكانيزمهاى دفاعى رئى

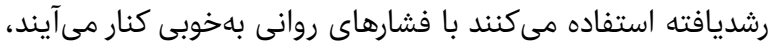

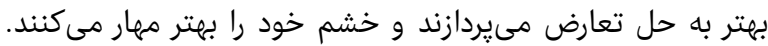

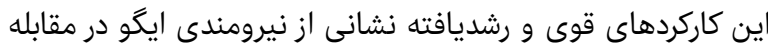

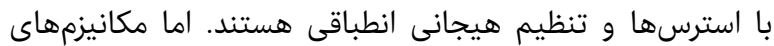

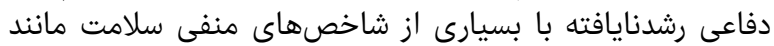

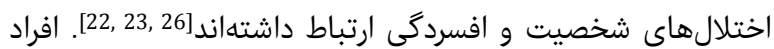

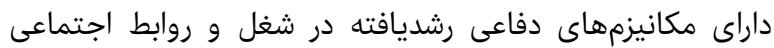

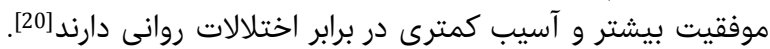

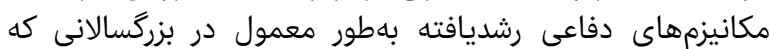

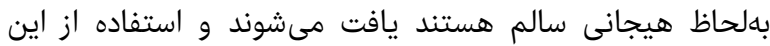

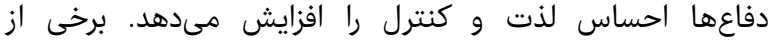

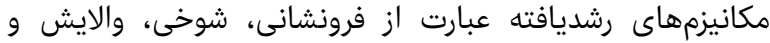

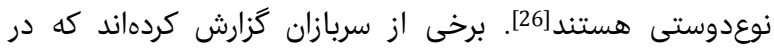

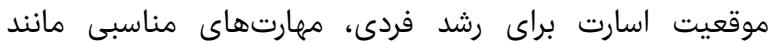

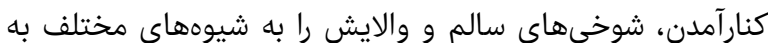

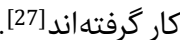

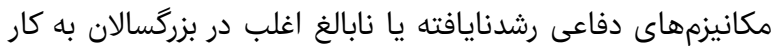

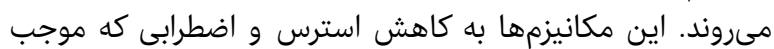

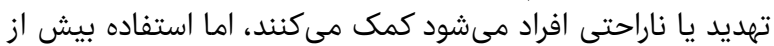

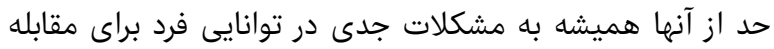

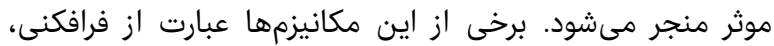

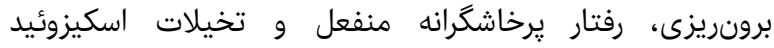

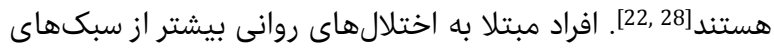

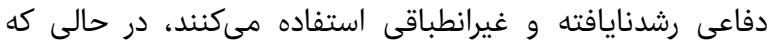

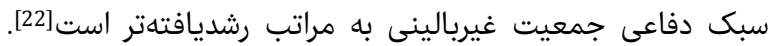

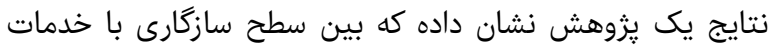

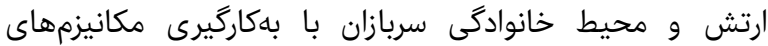

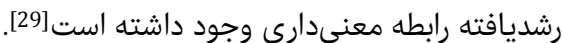

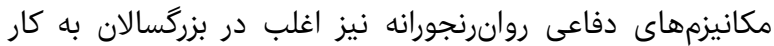

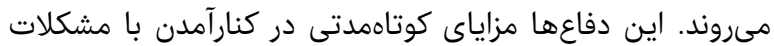

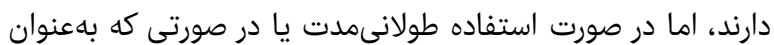

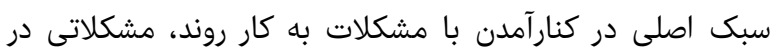

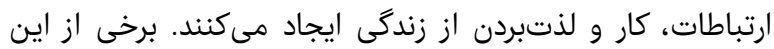

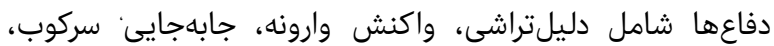

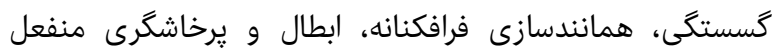

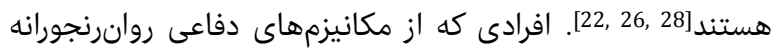

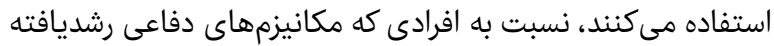

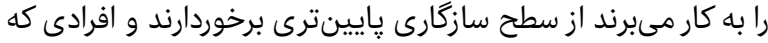

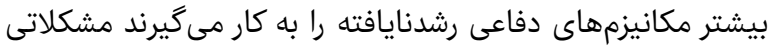

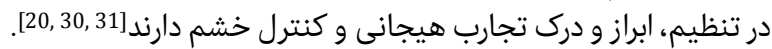

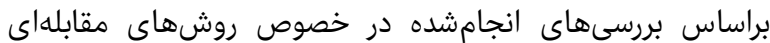

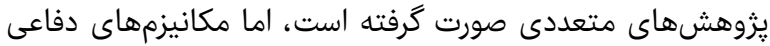

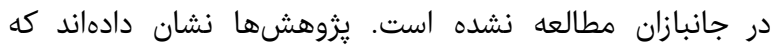

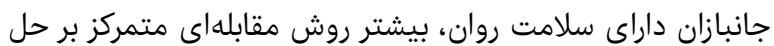

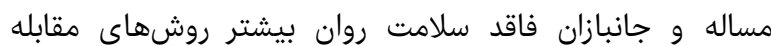

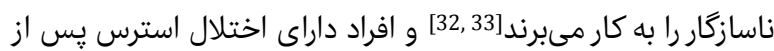

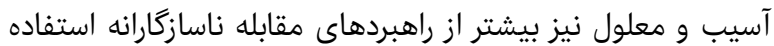

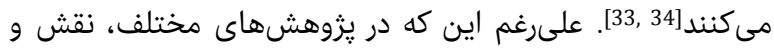

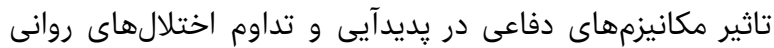

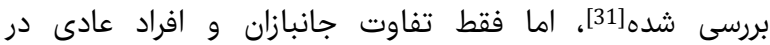
بهكارگيرى راهبردهاى مقابله ارادى مطالعه شده و مكانيان انيزمهاى 


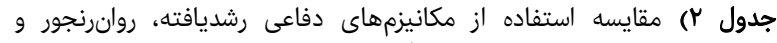

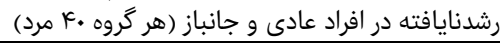

\begin{tabular}{|c|c|c|c|}
\hline سطح معنىدارى & Fيزان F مي & نمرات & مكانيزمهاى دفاعى \\
\hline \multirow{3}{*}{$<. / .1$} & \multirow{3}{*}{$|F / V| S$} & & رشديافته \\
\hline & & $\kappa \Delta / V \pm \Lambda \Delta / \Delta$. & عادى \\
\hline & & $\mu \Delta / \| \pm r V / V$. & جانباز \\
\hline \multirow{3}{*}{$<. / .1$} & \multirow{3}{*}{$r / V k \mu$} & & روانرنجورانه \\
\hline & & $\mu V / I V \pm V / \varepsilon q$ & عادى \\
\hline & & $k y / 11 \pm V V / 19$ & جانباز \\
\hline \multirow{3}{*}{$>.1 .0$} & \multirow{3}{*}{ ৭/ৎQ। } & & ر رشدنايافته \\
\hline & & $\|r /\| V \pm 9 \cdot \mid q r$ & 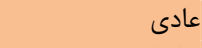 \\
\hline & & $\mid \Psi N / r \varepsilon \pm \varphi \Delta / \cdot K$ & جانباز \\
\hline
\end{tabular}

بحث بروث حاضر با هرف مقايس4 مكانيزمهاى دفاعى رثديافته،

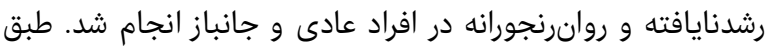

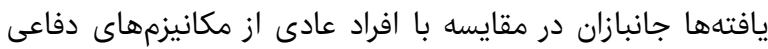

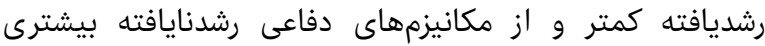

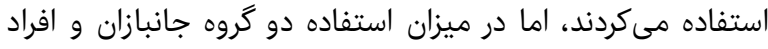

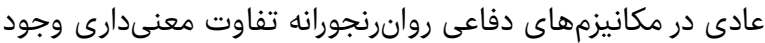

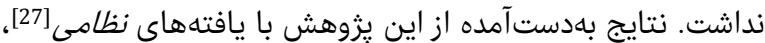

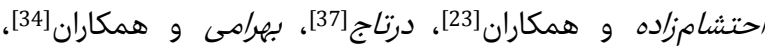

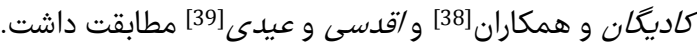

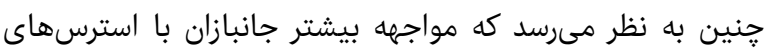

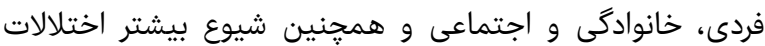

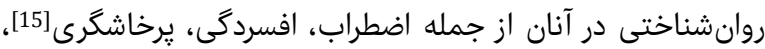

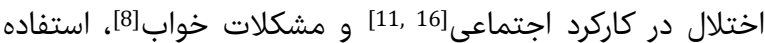

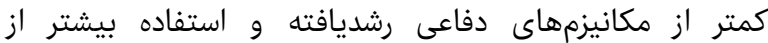

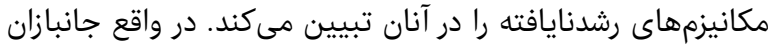

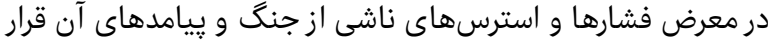

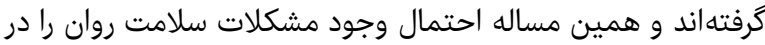

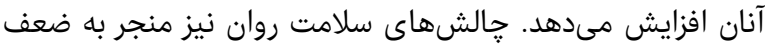

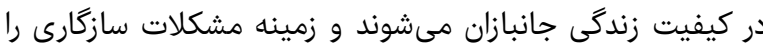

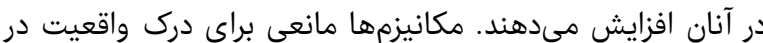

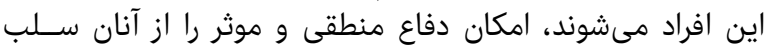

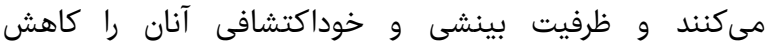

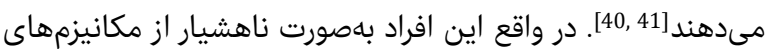

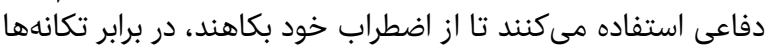

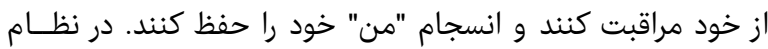

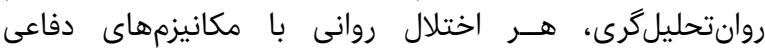

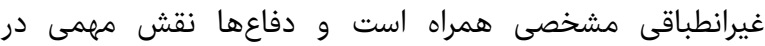

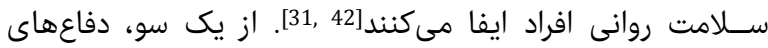

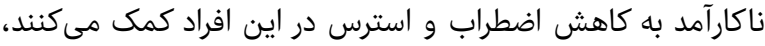

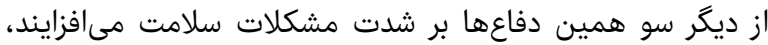

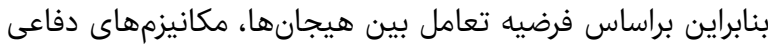

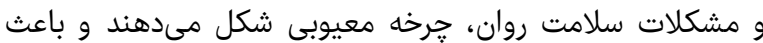

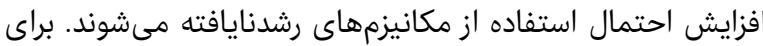

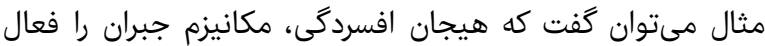

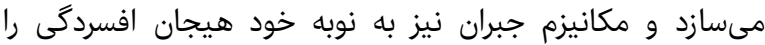

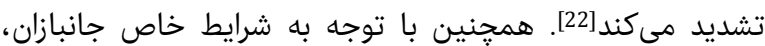

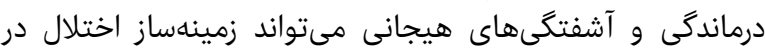

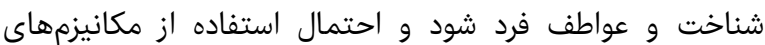
رشدنايافته را در شرايط استرسزا افزايش دهد فرد احتمال استفاده از مكانيزمهاي

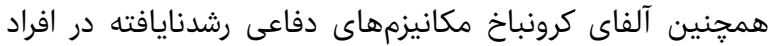

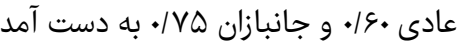

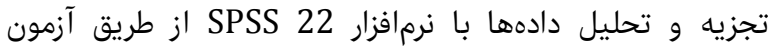

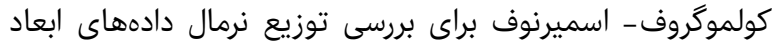

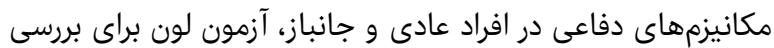

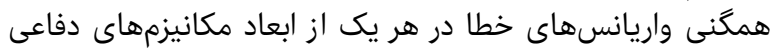

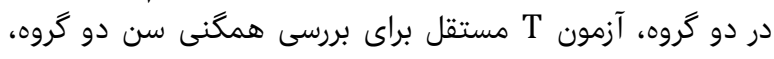

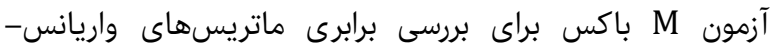

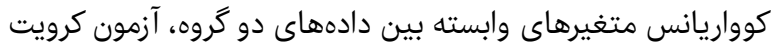

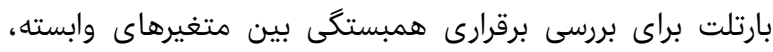

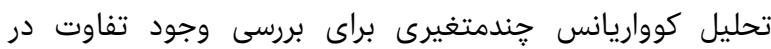

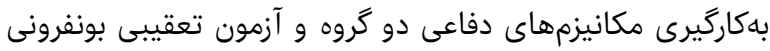

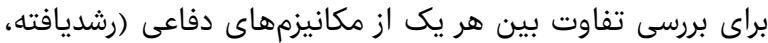

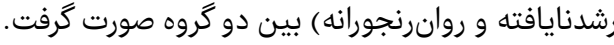

\section{يافتهها}

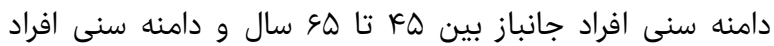

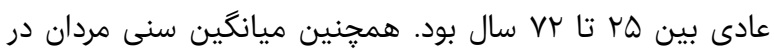
كروه جانبازان

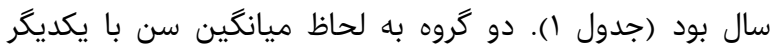

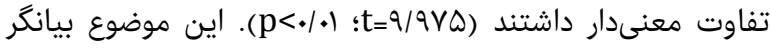

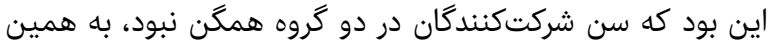

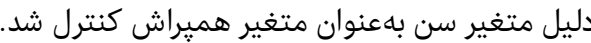

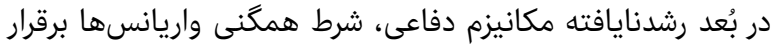

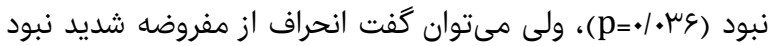

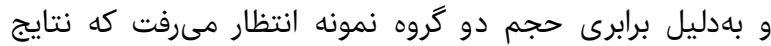
تحليل را بى بـاعتبار نسازد. با كنترل اثر سن، بين اعنبارنازد افراد عادى و جانباز در استفاده از انواع

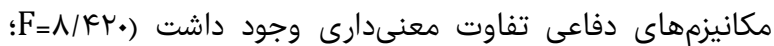

$(\mathrm{p}<\bullet / \cdot 1$

جدول () توزيع فراوانى مطلق و نسبى (اعداد داخل يرانتز، درصد هستند)

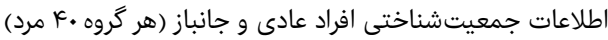

\begin{tabular}{|c|c|c|}
\hline جانبازان & افراد عادى & متغيرها \\
\hline & & سطح تحصيلات \\
\hline$\mu \cdot(V \Delta / \cdot)$ & $\Lambda(r \cdot / \cdot)$ & دييلم و پايينتر \\
\hline$r(\Delta / \bullet)$ & $\mu(V / Q)$ & كاردانى \\
\hline$k(1.1)$. & $1 Q\left(\mu^{\mu} \vee / \Delta\right)$ & كارشناسى \\
\hline$r(\Delta / \cdot)$ & $\mathbb{I}^{\mu}(\mu / / \Delta)$ & كارشناسى ارشد \\
\hline$r(\Delta / \cdot)$ & $I(r / Q)$ & دكترى \\
\hline & & وضعيت شغلى \\
\hline$\mu \mu(\Delta V / \Delta)$ & - & بيكار \\
\hline$q(r Y / Q)$ & $\mu V(q r / Q)$ & شاغل \\
\hline$\Lambda(r \cdot / \cdot)$ & $\mu(V / Q)$ & بازنشسته \\
\hline & & وضعيت تاهل \\
\hline$\varphi(|Q| \cdot)$ & $\wedge(r \cdot / \cdot)$ & مجرد \\
\hline$\mu_{\mathcal{K}}(\wedge \omega / \cdot)$ & $\mu r(\Lambda \cdot / \cdot)$ & متاهل \\
\hline
\end{tabular}

تفاوت ميانكين نمرات استفاده از بُعد رشديافته و رشدنايافته

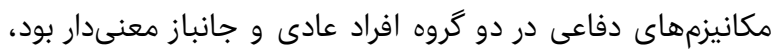

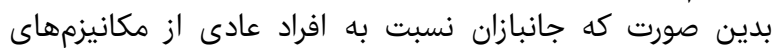

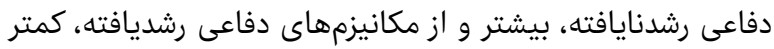

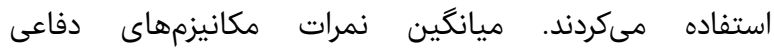

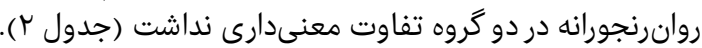




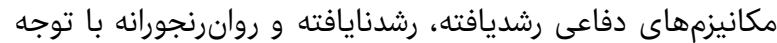

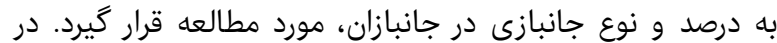

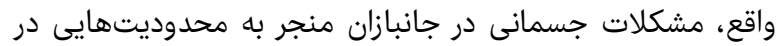

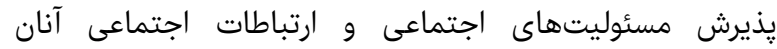

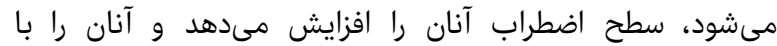

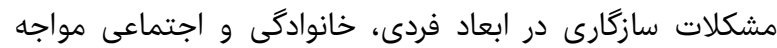

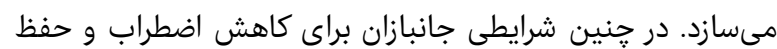

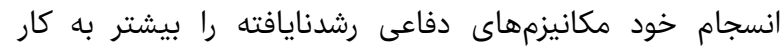

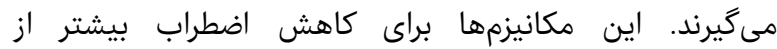

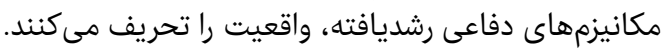

\section{نتيجه گليرى}

جانبازان از مكانيزمهاى دفاعى رشديافته كمتر و مكانيزمهاى

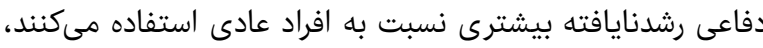

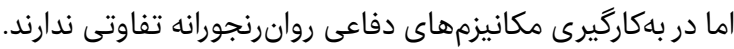

تشكر و قدردانى: مراتب سياس و قدردانى خود را از كليه افرادى كه

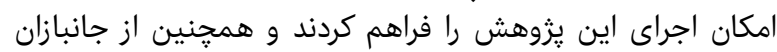

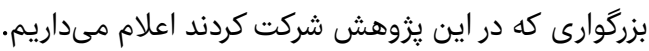

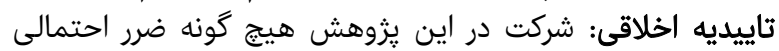

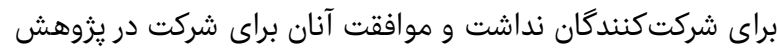

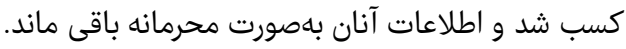

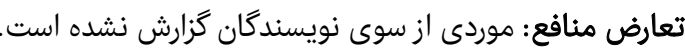

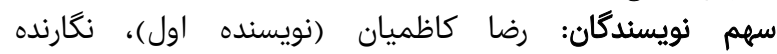

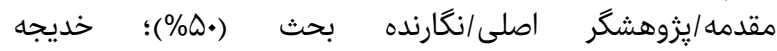

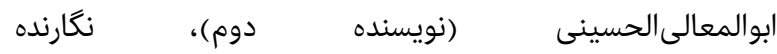

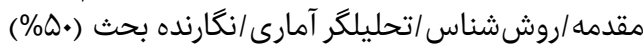

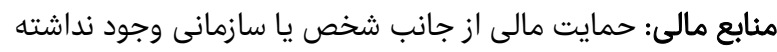
است.

منابع

1- Esmaeili L, Pourabaian Esfahani MH, Dabbashi F. Effect of music-therapy on depression of chronic PTSD veterans. Iran J War Public Health. 2015;7(2):73-9. [Persian]

2- Ghafouri Fard M, Payami Bousari M, Heydari Soomee AR. A survey on various dimensions of adjustment status among warfare Veterans with one eye blindness. Iran J War Public Health. 2014;6(2):64-71. [Persian]

3- Mc Kinney JM, Hirsch JK, Britton PC. PTSD symptoms and suicide risk in veterans: Serial indirect effects via depression and anger. J Affect Disord. 2017;214:100-7.

4- Rieson MR, Miri MR, Dastjerdi R, Sharifzadeh GR. Prevalence of mental disorders in Birjand-2010. J Birjand Univ Med Sci. 2012;19(1):81-7. [Persian]

5- Khani H, Zamani N, Ghorbani A, Jahangir AH, Jena Abadi H. Mental health status of amputee veterans Mazandaran province, Iran. Iran J War Public Health. 2016;8(3):149-56. [Persian]

6- Hasani Tabatabai L, Shaker Dioulagh A. Comparison of stress and social support between veterans and nonveterans, case study of Urmia city, Iran. Iran J War Public Health. 2017;9(3):141-6. [Persian]

7- Mandani B, Fakhri A. Study of health related quality of life in posttraumatic stress disorder war veterans. Iran J War Public Health. 2013;5(2):18-25. [Persian]

8- Vinnikov D, Blanc PD, Alilin A, Zutler M, Holty JEC. Fatigue and sleepiness determine respiratory quality of
دفاعى رشدنايافته به سبك دفاعى غالب فرد تبديل شوند، منجر به

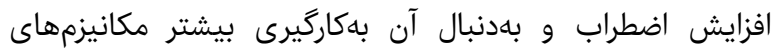
دفاعى مىشوند[31].

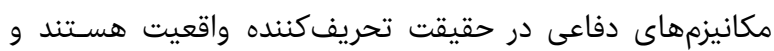

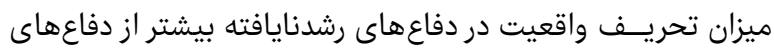

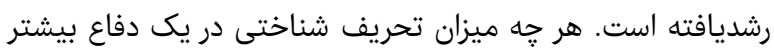

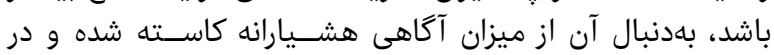

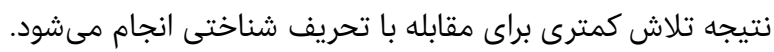

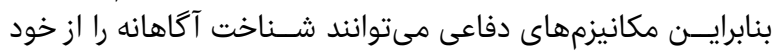

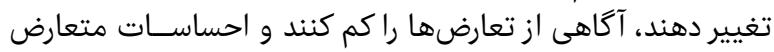

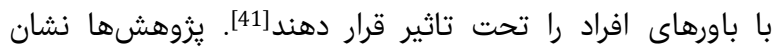

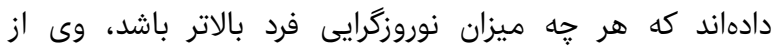

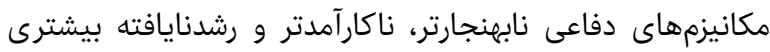

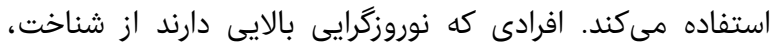

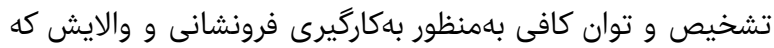

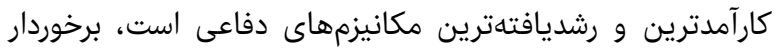

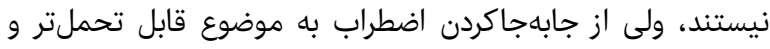

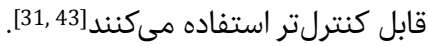

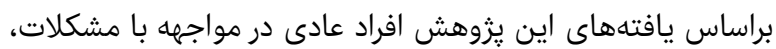

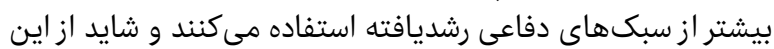

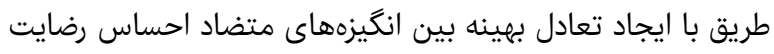

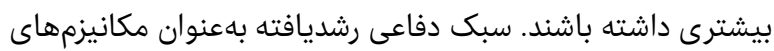

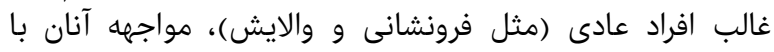

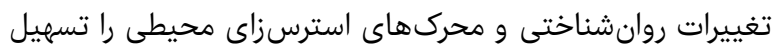

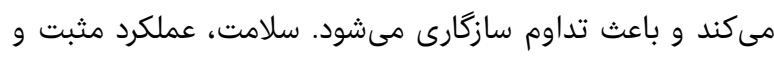

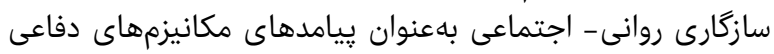

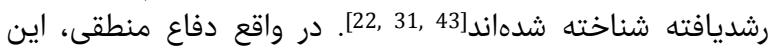

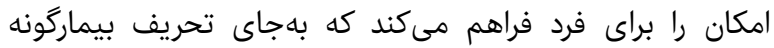

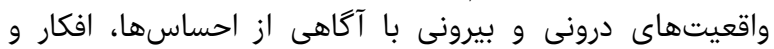

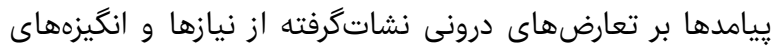

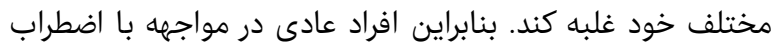

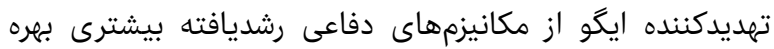

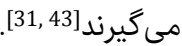
يافته ديكر اين بود كه بين جانبازئن

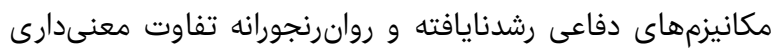

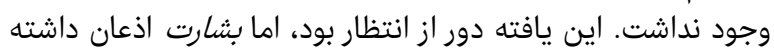

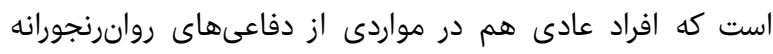

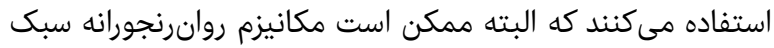

غالب افراد عادى نباشد [31].

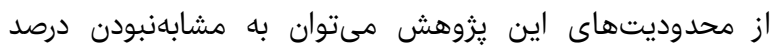

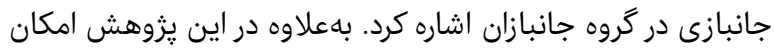

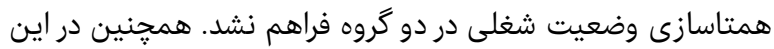

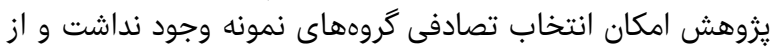

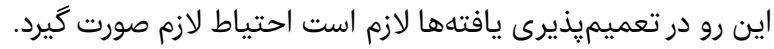

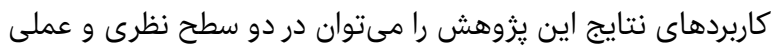

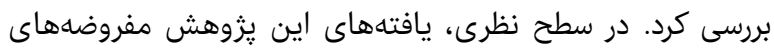

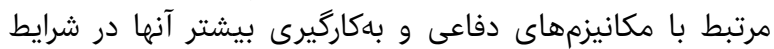

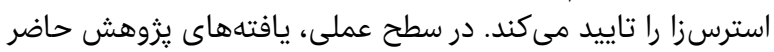

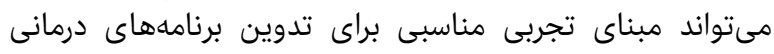

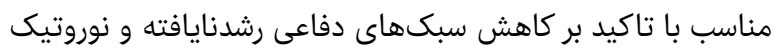

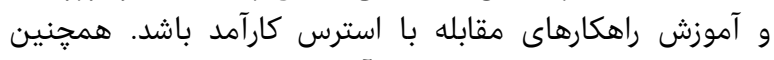

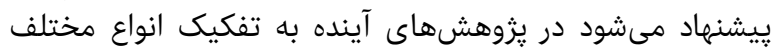


mechanisms with stress with mental health in war veterans of Zahedan in the academic year of 2002-2003 [Dissertation]. Zahedan: Zahedan University of Medical Sciences; 2003. [Persian]

28- Andrews G, Singh M, Bond M. The defense style questionnaire. J Nerv Ment Dis. 1993;181(4):246-56.

29- Dedic G. Defense mechanisms in soldiers during the period of adaptation to the military environment. Vojnosanit Pregl. 2000;57(4):393-401. [Serbian]

30- Zoccali R, Muscatello MRA, Bruno A, Cedro C, Campolo D, Pandolfo G, et al. The role of defense mechanisms in the modulation of anger experience and expression: Gender differences and influence on selfreport measures. Personal Individ Differ. 2007;43(6):1426-36.

31- Besharat MA. Defense mechanisms in patients with major depression, anxiety disorders, and normal individuals: A comparative study. Res Clin Psychol Couns. 2013;3(1):41-54. [Persian]

32- Aflakseir A. The role of social support and coping strategies on mental health of a group of Iranian disabled war veterans. Iran J Psychiatry. 2010;5(3):102-7.

33- Pietrzak RH, Harpaz-Rotem I, Southwick SM. Cognitive-behavioral coping strategies associated with combat-related PTSD in treatment-seeking OEF-OIF veterans. Psychiatry Res. 2011;189(2):251-8.

34- Bahrami S, Vaez Mousavi MK, Davari F. The level and type of irrational beliefs among athletes in the national disabled athletics team. Middle East J Disabil Stud. 2014;4(2):26-32. [Persian]

35- Delavar A. Theoretical and practical foundations of research in humanities and social sciences. Tehran: Rooshd; 2017. [Persian]

36- Heidari Nasab L, Mansour M, Azade Fallah P, Shoeyri MR. Validity and reliability of the defensive styles questionnaire in Iranian samples. Daneshver Raftar. 2007;14(22):11-26. [Persian]

37- Dortaj F. Epidemiology of substance abuse in war survivors and their relatives. Iran J War Public Health. 2009;1(3):46-59. [Persian]

38- Cadigan JM, Klanecky AK, Martens MP. An examination of alcohol risk profiles and co-occurring mental health symptoms among OEF/OIF veterans. Addict Behav. 2017;70:54-60.

39- Aqdasi AN, Eydi R. A study of the relationship between mental health and confronting procedures among the spinal cord. J Instruct Eval. 2011;4(16):51-67. [Persian]

40- Afzali MH, Fathi Ashtiani A, Azad Fallah P. Comparison of defense styles \& defense mechanisms of patients with obssesive-compulsive disorder \& normal people. J Behav Sci. 2008;2(3):245-52. [Persian]

41- Milliken CS, Auchterlonie JL, Hoge CW. Longitudinal assessment of mental health problems among active and reserve component soldiers returning from the Iraq war. JAMA. 2007;298(18):2141-8.

42- Besharat MA, Shahidi Sh. What is the relationship between alexithymia and ego defense styles? A correlational study with Iranian students. Asian J Psychiatry. 2011;4(2):145-9.

43- Zandi S, Shahabi Nejad Z, Borhan A. Predicting defense mechanisms based on big five personality traits among university students. Zanco J Med Sci. 2017;18(56):21-32. [Persian] life among veterans evaluated for sleep apnea. Health Qual Life Outcomes. 2017;15:48.

9- Jafari F, Gity Navard F, Soroush MR, Mousavi B. Quality of life in chemical war victims with sever pulmonary damage. Iran J War Public Health. 2012;4(1):46-52. [Persian]

10- Gholipour S, Parhizgar Kalat SZ, Gholipour M. Type of injury and quality of life among veterans with disabilities of Kermanshah province. Soc Work Res J. 2017;1(4):6699. [Persian]

11- Christensen J, Ipsen $\mathrm{T}$, Doherty $\mathrm{P}$, Langberg $\mathrm{H}$. Physical and social factors determining quality of life for veterans with lower-limb amputation(s): A systematic review. Disabil Rehabil. 2016;38(24):2345-53.

12- Yavari A, Esfandiari E, Masoumi M, Saeedi H, Allami M. Consideration of chronic pain and current prosthetic satisfaction in above knee amputees. Iran J War Public Health. 2011;3(2):53-8. [Persian]

13- Shahriar Sh, Masoumi M, Mousavi B, Soroush MR. Genitourinary disease in war related Bilateral lower limb amputation. Iran J War Public Health. 2009;1(3):17-24. [Persian]

14- Manaei Z, Abolmaali Alhossein Kh, Mousavi B. Epidemiology of mental health status of veterans with monocular blindness, case study of Fars province. Iran J War Public Health. 2016;8(4):235-41. [Persian]

15- Khodayarian M, Vaezi A, Jalapour S, Jalalpour T, Haji Hosseini $\mathrm{H}$. The survey of quality of life in spinal cord injured people in Yazd in 2014. J Mil Caring Sci. 2015;1(2):88-98. [Persian]

16- Walburg V, Chiaramello S. Link between early maladaptive schemas and defense mechanisms. Eur Rev Appl Psychol (Revue Européenne de Psychologie Appliquée). 2015;65(5):221-6. [French]

17- Valiente C, Swanson J, Eisenberg N. Linking students' emotions and academic achievement: When and why emotions matter. Child Dev Perspect. 2012;6(2):129-35.

18- Offer R, Lavie R, Gothelf D, Apter A. Defense mechanisms, negative emotions, and psychopathology in adolescent inpatients. Compr Psychiatry. 2000;41(1):3541.

19- Blackman JS. 101 Defenses: How the mind shields itself. Hove: Psychology Press; 2004.

20- Malone JC, Cohen S, Liu SR, Vaillant GE, Waldinger RJ. Adaptive midlife defense mechanisms and late-life health. Pers Individ Dif. 2013;55(2):85-9.

21- Corey G. Theory and practice of counseling and psychotherapy. Boston: Cengage Learning; 2017.

22- Cramer P. Defense mechanisms in psychology today, further processes for adaptation. Am Psychol. 2000;55(6):637-46.

23- Ehtesham Zadeh P, Pasha R, Samimi Z. The comparison of defense mechanisms of the production personnel, repair parts (direct revival) and administrative staff of Khuzestan steel company. J Soc Psychol (New Find Psychol). 2012;7(24):21-33. [Persian] 24- Carlson NR. Psychology: The science of behavior. London: Pearson Education; 2010.

25- Vaillant GE. Adaptive mental mechanisms, their role in a positive psychology. Am Psychol. 2000;55(1):89-98.

26- Schacter D, Gilbert D, Wegner D, Hood B. Psychology: Second European edition. London: Macmillan International Higher Education; 2015.

27- Nezami H. Comparison of the type of coping 